\title{
Characterization of infrared surface plasmon resonances generated from a fiber-optical sensor utilizing tilted Bragg gratings
}

\author{
T. Allsop, ${ }^{1, *}$ R. Neal, ${ }^{2}$ S. Rehman, ${ }^{3}$ D. J. Webb, ${ }^{1}$ D. Mapps, ${ }^{2}$ and I. Bennion ${ }^{1}$ \\ ${ }^{1}$ Photonics Research Group, Aston University, Aston Triangle, Birmingham, B4 7ET, United Kingdom \\ ${ }^{2}$ Department of Communications and Electrical Engineering, Facility of Technology, University of Plymouth, \\ Plymouth, PL4 8AA, United Kingdom \\ ${ }^{3}$ FiberLogix Limited, Ashley House, Vale Industrial Park, Tolpits Lane, Watford, Herts, WD18 9QP, United Kingdom \\ *Corresponding author: t.d.p.allsop@aston.ac.uk
}

Received August 16, 2007; revised November 28, 2007; accepted January 18, 2008; posted January 30, 2008 (Doc. ID 86532); published March 17, 2008

\begin{abstract}
We demonstrate the use of tilted fiber gratings to assist with the generation of infrared surface plasmons on a metal film coating the flat of a D-shaped fiber. The wavelength of the strong $(>25 \mathrm{~dB})$ resonance is tunable over $\sim 1000 \mathrm{~nm}$ by adjusting the polarization state of the light and is highly sensitive to the refractive index of any aqueous medium surrounding the fiber (sensitivity=3365 nm). (C) 2008 Optical Society of America

OCIS codes: $060.2370,060.2310$.
\end{abstract}

\section{INTRODUCTION}

Gratings in fibers-including long period gratings (LPGs), fiber Bragg gratings (FBGs), and tilted FBGs (TFBGs)and surface plasmon resonances (SPRs) (in both planar and fiber configurations) have found many potential uses in diverse applications, such as telecommunications and environmental sensing. In optical communications, a common use for fiber grating devices is spectral filtering [1] while fiber SPR devices can be used as polarizers [2]. In the field of environmental sensing both sets of devices can used to detect changes in the refractive index of various substances, so there are potential applications in chemistry, biochemistry, and biology [1,3-5]. SPR is an important optical phenomenon that involves a resonant transfer of the pumping light energy to a surface-plasmon (SP) mode in the form of collective oscillations of electrons at the surface of a metal [6]. It has been found that SPR generation is very sensitive to the polarization of the illuminating light, its wavelength, the angle of incidence on the metal surface, and the refractive index of the media adjacent to the metal surface, which is important for monitoring biochemical/chemical reactions [7]. SPR biosensors offer the opportunity for real-time and label-free monitoring of biomolecular interactions [8]. The majority of SPR based systems operate in the visible or near infrared part of the spectrum which gives a probe depth (the spatial extension of the SP into the environment beyond the surface of the metal) of $\sim$ from 200 to $300 \mathrm{~nm}$ [7], though going to longer wavelengths may improve sensor performance [6] and reduce costs by making use of standard telecommunications components. The plasmons obey the following dispersion relation for two homogeneous semi-infinite media [9]:

$$
\beta=k \sqrt{\left(\frac{\epsilon_{m} \cdot n_{\mathrm{s}}^{2}}{\epsilon_{m}+n_{\mathrm{s}}^{2}}\right)},
$$

where $k$ is the free space wave number, $\epsilon_{m}$ is the dielectric constant of the metal $\left(\epsilon_{m}=\epsilon_{m r}+i \epsilon_{m i}\right)$, and $n_{s}$ is the refractive index of the sample to be tested, placed in contact with the far surface of the metal.

Generally fiber gratings comprise an axially periodic refractive index variation inscribed in the core of a photosensitive single-mode optical fiber by ultraviolet radiation, or other means, that couples light from the core to other core modes (FBG), to cladding modes (LPGs), or to some combination of core, cladding, and radiation modes (TFBGs) at discrete wavelengths [9]. There has been a lot of work published with regard to fiber grating based devices for refractive index sensing; with the majority of the these devices, the highest index sensitivity is obtained with test sample indices over $\sim 1.4$ [10]. They generally have significantly less sensitivity in the aqueous index regime, which is important for biochemical sensing.

In this paper, we report on what we believe is a novel SPR fiber device utilizing a TFBG to enhance the coupling of the illuminating light to a SP generated on a silver coating on a lapped single mode fiber $[11,12]$. It was observed that the spectral location of maximum coupling to the SPR was highly dependent upon the polarization state of the illuminating light and could be tuned from 1100 to $1700 \mathrm{~nm}$, thus producing various SP probe depths, dependent upon the polarization. It was found that for a device that is still to be fully optimized, the maximum spectral index sensitivity $(\mathrm{d} \lambda / \mathrm{d} n)$ was $3365 \mathrm{~nm}$ for the index range from 1.335 to 1.370 (suitable for refractive index monitoring of aqueous solutions) for SPs generated 
from 1200 to $1700 \mathrm{~nm}$. Modeling the operation of this device provided reasonable agreement with experimental results. Our results suggest that with an optimized fiber device, at least another order of magnitude increase in the sensitivity should be possible.

\section{FABRICATION AND CHARACTERIZATION}

The fiber devices are constructed in three stages. First, the TFBG is written in a UV photosensitive single-mode fiber (hydrogenated standard telecommunications fiber) using a uniform phase mask (mask period $=1.0157 \mu \mathrm{m}$ ) mounted on a goniometer and then tilted to a specific angle; labels are added to indicate the orientation of the tilted grating. Second, the fiber is lapped down to $10 \mu \mathrm{m}$ from the core-cladding interface; it has been estimated that the error associated with this lapping is approximately $\pm 1 \mu \mathrm{m}$. The labels on the fiber are used to indicate which region of cladding is to be removed so that the grating vector, the fiber axis, and the normal to the lapped surface all lie in a plane. Third, the flat of the lapped fiber is then coated with a $35 \mathrm{~nm}$ layer of silver using a sputter machine and mask. The lapping of the fiber induced a general loss of 4-6 dB at the longer wavelength side of the Bragg resonance with an extra loss of $3 \mathrm{~dB}$ on the short wavelength side, see Fig. 1 . After the silver coating was applied, there was an additional general loss observed ranging from 5 to $17 \mathrm{~dB}$. This loss range may be expected due to small variations in the lapping depth, which can change the coupling efficiency dramatically. Furthermore there are likely to be variations in the precise shape of the side polished region itself, which again can affect the loss of the devices.

The fiber devices were characterized with a broadband light source that was passed through a polarizer, a polarization controller, and a polarization maintaining coupler before illumination of the sample. The transmission spectra were monitored by using an optical spectrum analyzer (OSA) with a resolution of $0.005 \mathrm{~nm}$, and the polarization of the illuminating light via the coupler, using a polarimeter. The polarization was monitored in this way, rather than at the connector just before the device, because the latter approach would involve disconnecting the sensor prior to each polarization measurement and this would likely induce greater polarization changes than we were trying to measure (see Fig. 2).

A series of these devices was fabricated with angles of tilt from $1^{\circ}$ to $9^{\circ}$. It was found that it was possible to obtain SPRs with these devices over a wide range of wavelengths, by tuning the polarization properties of the illuminating light, see Fig. 3. Note that the features seen at maximum coupling of the SPR are artifacts due to the normalization procedure of the OSA and that the transmission minimum of the resonance can be considerably narrower. The polarization dependency of the devices was investigated using the arrangement shown in Fig. 2. The devices were submerged into various index solutions and the ellipticity and azimuth of the polarization of the illuminating light was changed. An example of the dependency is shown in Fig. 4. Note that with the arrangement used, we cannot rely on the polarization at the device being identical to that measured by the polarimeter; consequently, Fig. 4 is intended to demonstrate the strong sensitivity of coupling strength and resonant wavelength to polarization, without implying any quantitative relationship.

An inspection of Fig. 4 shows that the maximum extinction induced by the coupling to the SP is very much dependent upon the polarization of the illuminating light. The example shown relates to azimuth but this was also seen for ellipticity. It is expected that the coupling efficiency and spectral location of the resonance should dramatically change with polarization, with some polarization states resulting in low coupling efficiencies of only a few decibels [10]. However, while variations in the coupling strength with polarization were observed, these devices still produced large extinction ratios over a very wide wavelength range, see Fig. 1 . In Fig. 3 it can be seen that over the observed spectral range $(1220-1700 \mathrm{~nm})$, the $7^{\circ}$ tilt angle device in a solution with a refractive index of 1.360 exhibited extinction ratios in excess of $35 \mathrm{~dB}$. It can also be seen from Fig. 3 that the extinction range varies from $\sim 1$ to $35 \mathrm{~dB}$ for a given wavelength, as a func-
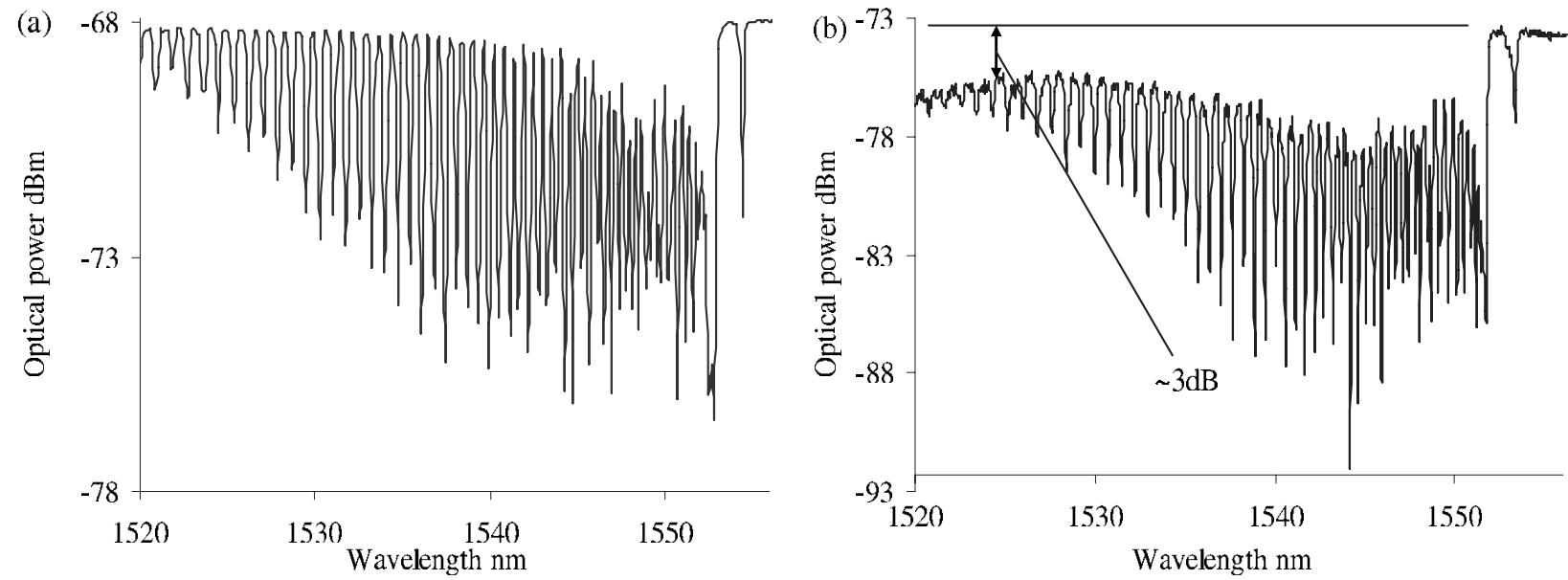

Fig. 1. Comparison of transmission spectra using nonpolarized light from a tilted Bragg grating (length: $3.2 \mathrm{~cm}$, tilt: $3^{\circ}$ ) before (a) and after lapping (b). 


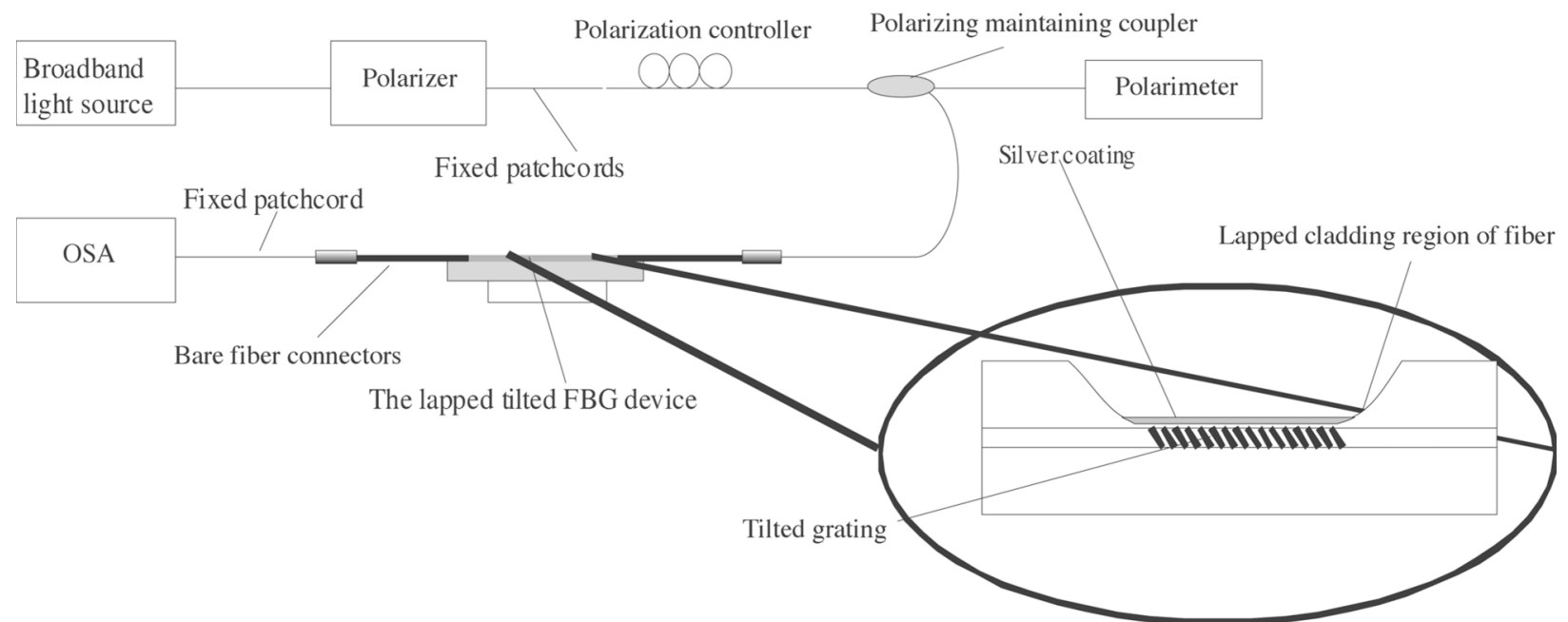

Fig. 2. Scheme used for the characterization of the tilted, lapped, and coated fiber Bragg grating device.

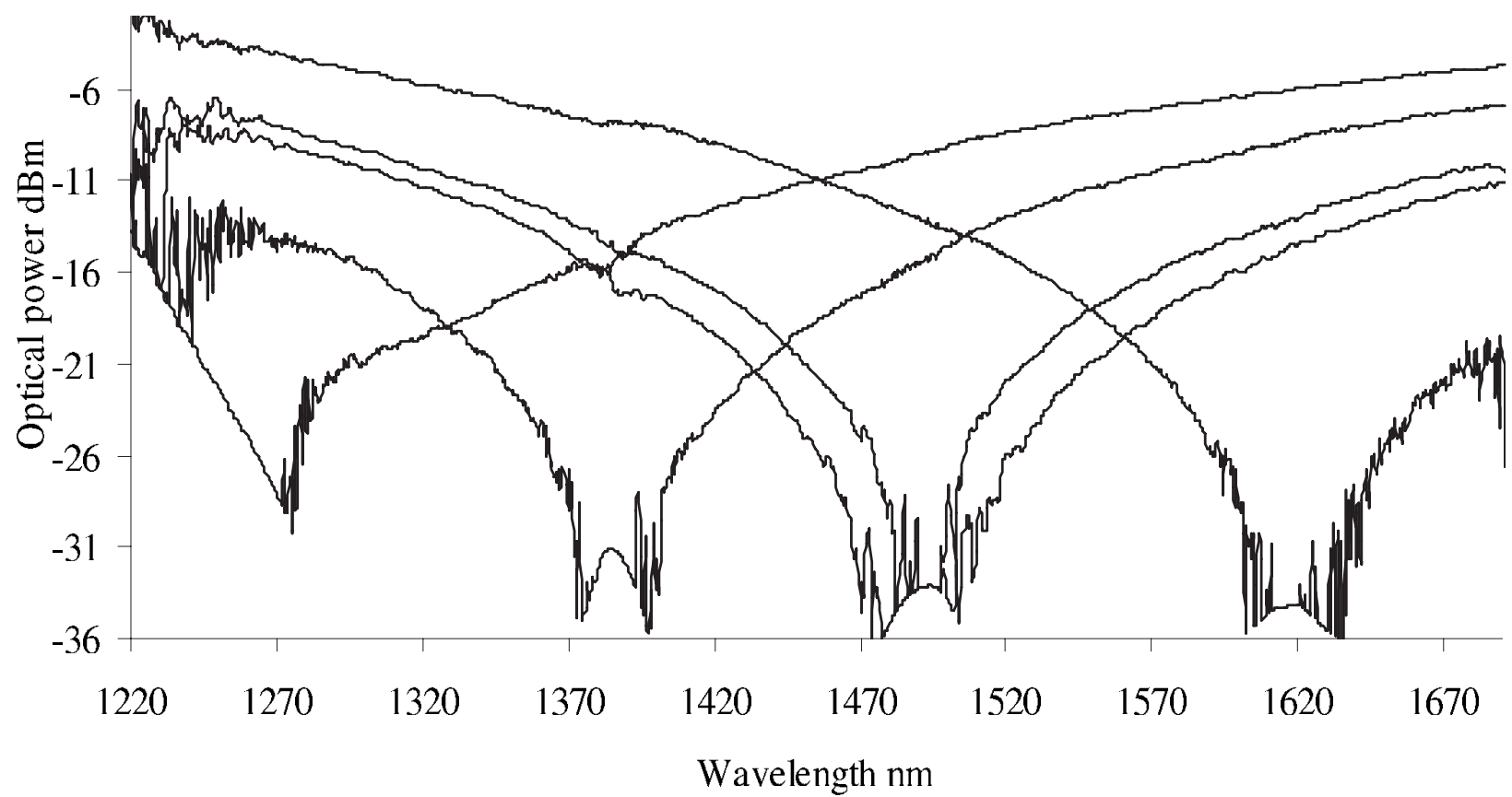

Fig. 3. Normalized transmission spectra of a fiber SPR device with a tilt angle of $7^{\circ}$ and length $2.2 \mathrm{~cm}$ immersed in a solution with an index of 1.360 and illuminated with light with various polarization states.

tion of polarization. Figure 4 demonstrates that the maximum extinction ratio occurs with a surrounding medium with a refractive index of $\sim 1.36$ with some reduction for both lower and higher refractive indices.

The refractive index sensitivity was further investigated. The $7^{\circ}$ device was placed in a V-groove and immersed in certified refractive index (CRI) liquids (supplied by Cargille Laboratories, Inc.) which have a quoted accuracy of \pm 0.0002 . The fiber and V-groove were carefully cleaned, washed in ethanol and then in deionized water, and finally dried before the immersion of the device into the next CRI liquid. The V-groove was made in an aluminium plate, machined flat to minimize bending of the fiber, and the plate was placed on an optical table, which acted as a heat sink to maintain a constant tem- perature. The fiber device was used in conjunction with the apparatus shown in Fig. 2. An example of the spectral response of one device as a function of the surrounding medium's refractive index at a fixed polarization state is shown in Fig. 5. It was also found that changing the tilt angle of the FBG changed the maximum extinction ratio achievable by altering the polarization of the illuminating light, as shown in Fig. 6.

By comparing the coupling strength to the SP with and without a grating (Fig. 6), it can be seen that the grating greatly enhances the coupling in the aqueous index regime from $\sim 4 \mathrm{~dB}$ without grating to $25 \mathrm{~dB}$ for the $7^{\circ}$ tilted grating, with coupling increasing with increasing surrounding index to in excess of $35 \mathrm{~dB}$ over indices of 1.36. It was also observed that spectral features in the fiber de- 


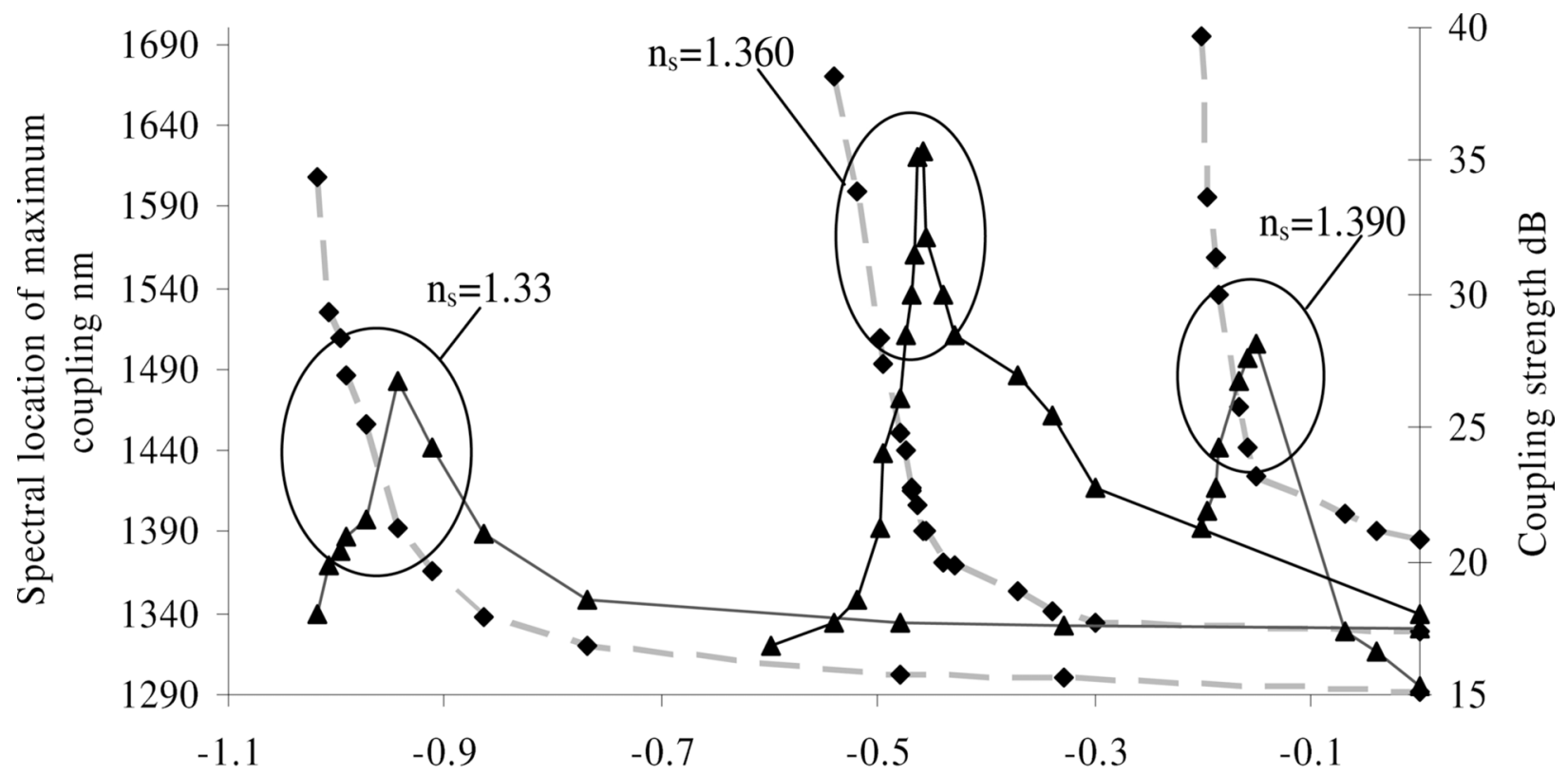

Change in polarization azimuth degrees

Fig. 4. Azimuthal polarization dependency of the SPR device (Ag thickness $35 \mathrm{~nm}$, tilt angle $3^{\circ}$, grating length $3.2 \mathrm{~cm}$ ), showing resonant wavelength (diamonds) and coupling strength (triangles) as a function of polarization for different refractive indices of the surrounding medium.
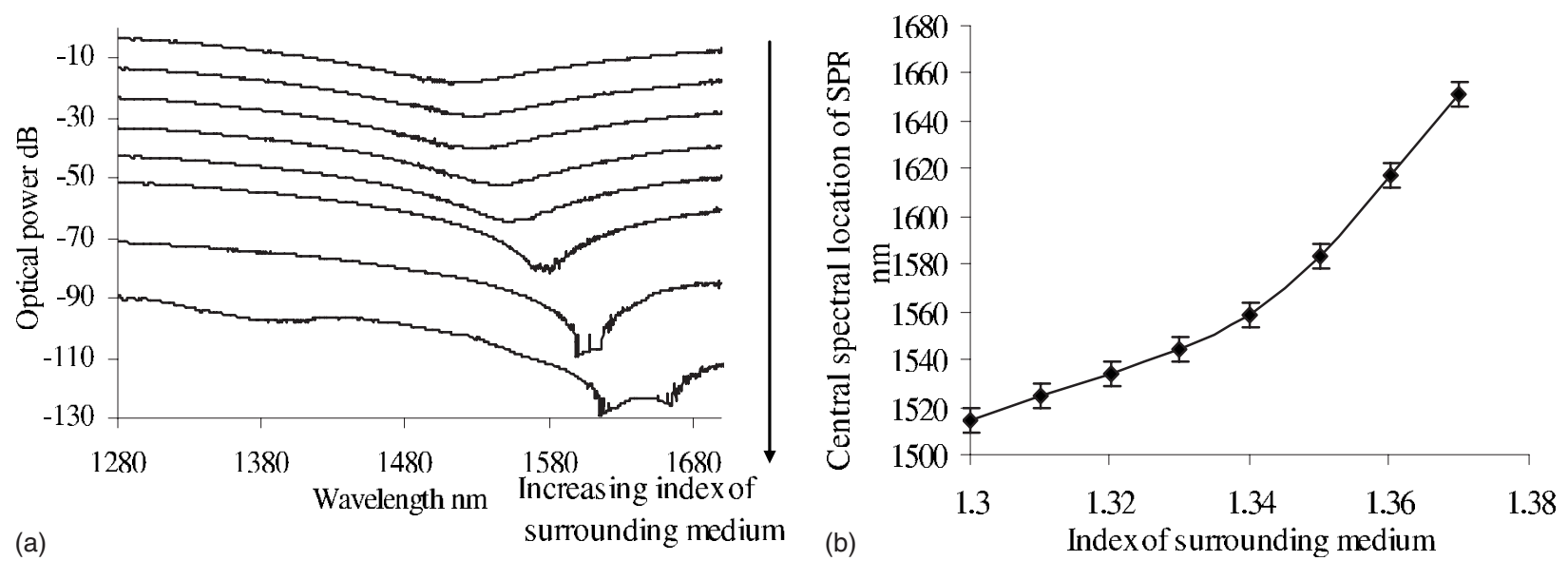

Fig. 5. (a) Transmission response and (b) shift in resonant wavelength as a function of the surrounding medium's refractive index for a given polarization state. Tilt angle $=7^{\circ}$, length $=2.2 \mathrm{~cm}$.

vice with no grating were very much broader than those with the gratings present. The spectral sensitivities obtained in the aqueous index regime and shown in Fig. 6(a) are comparable to the highest sensitivities obtained with SPR based devices [4]. The highest spectral sensitivity obtained was $\mathrm{d} \lambda / \mathrm{d} n=3365 \mathrm{~nm}$ from the $9^{\circ}$ tilt angle device leading to a resolution (under the assumption of a $0.1 \mathrm{~nm}$ measurement resolution for the resonance wavelength) of $\sim 2 \times 10^{-5}$ over the index range from 1.34 to 1.38 . For the fiber devices investigated to date, the spectral sensitivities varied from 700 to $1400 \mathrm{~nm}$ over the index regime from 1.3 to 1.34 , and from 2100 to $3400 \mathrm{~nm}$ over index regime from 1.34 to 1.38 . The power variation yielded results from 106 to $300 \mathrm{~dB}$ over the index regime from 1.3 to 1.34, and from 250 to $730 \mathrm{~dB}$ over the index regime from 1.34 to 1.38 . This sensor achieves stronger coupling to the
SP than other tilt angles, with strengths varying from 10 to $+30 \mathrm{~dB}$ in the aqueous index regime.

\section{MODELING AND PHYSICAL PROPERTIES}

A model was produced for these SPR fiber devices by first calculating the scattering angles associated with the propagation constants of the various transverse modes (TE/TM) of a D-shaped fiber with a silver coating. The scattering angle $(\alpha)$ is calculated from the effective index of the cladding modes $\left(n_{\beta}\right)$ by the relationship given by the ray approach; $\cos (\alpha)=n_{\beta} / n_{\mathrm{cl}}$, where $n_{\mathrm{cl}}$ is the refractive index of the cladding, this angle being to the fiber axis. These angles are used to give an associated incidence angle $(\varphi)$ of each cladding mode onto the metal/ dielectric interface and thus the cladding mode wave- 

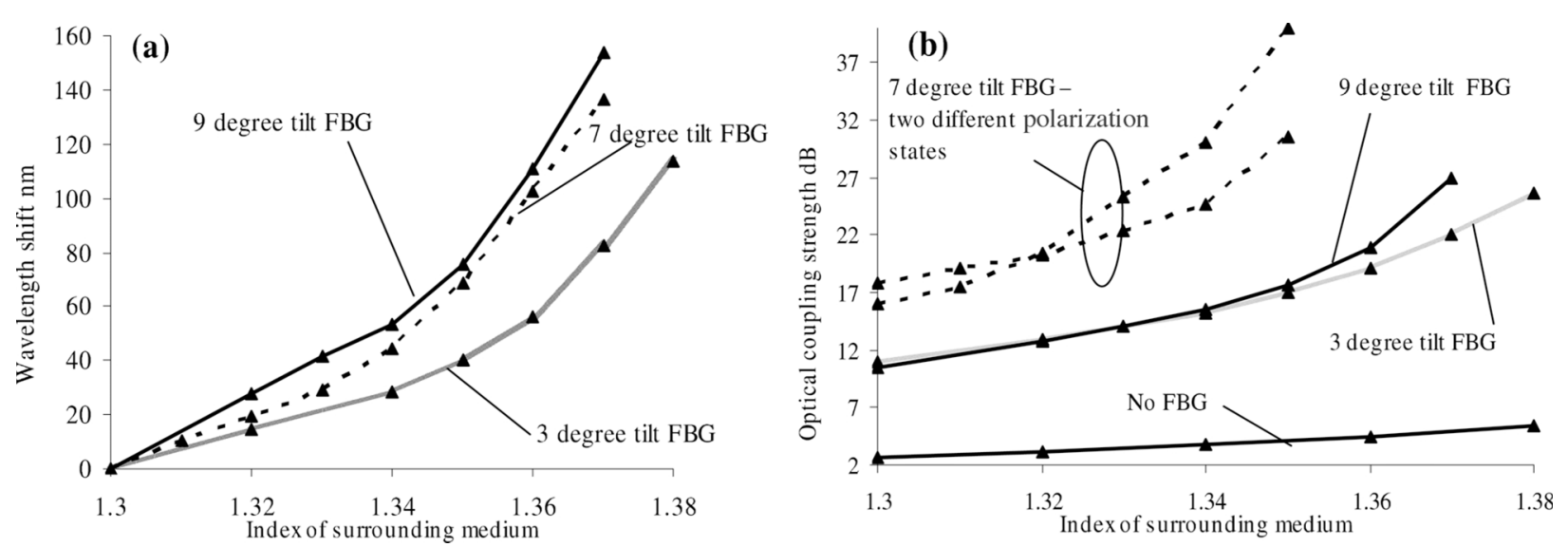

Fig. 6. Spectral characteristics of three devices with $3^{\circ}, 7^{\circ}$, and $9^{\circ}$ tilt angles and lengths $3.3,2.2$, and $1.7 \mathrm{~cm}$, respectively. (a) Wavelength dependence and (b) optical coupling strength dependence of the device as a function of the surrounding medium's refractive index. Also shown as a control in (b) is the coupling of a coated and lapped fiber with no grating inscribed.

number projection onto that interface. SPs are generated when this wave-number projection satisfies the dispersion relation of the plasmons given by expression (1), thus

$$
\frac{2 \pi}{\lambda} \sqrt{\left(\frac{\epsilon(\lambda)_{m} \cdot n(\lambda)_{s}^{2}}{\epsilon(\lambda)_{m}+n(\lambda)_{s}^{2}}\right)}=\frac{2 \pi \cdot n_{\mathrm{cl}}}{\lambda} \sin (\varphi) .
$$

The theoretical spectral transmission response of the SPR fiber device is obtained by calculating the reflected intensity at various wavelengths. The quantitative description of the minimum of the reflected intensity $R$ for an SPR can be obtained from Fresnel's equations for a three layered system. The reflectivity $R$ for $p$-polarized light, with $E_{0}{ }^{p}$ as the incoming and $E_{r}{ }^{p}$ as the reflected field, is given by [9] for a smooth surface:

$$
R=\left|\frac{E_{r}{ }^{p}}{E_{0}{ }^{p}}\right|^{2}=\left|\frac{r_{n_{2} n_{m}}{ }^{p}+r_{n_{m} n_{s}}{ }^{p} \cdot \exp \left(2 i \mathbf{K}_{z n_{m}} d\right)}{1+r_{n_{2} n_{m}}{ }^{p} \cdot r_{n_{m} n_{s}}{ }^{p} \cdot \exp \left(2 i \mathbf{K}_{z n_{m}} d\right)}\right|^{2},
$$

where $d$ is the thickness of the metal coating and $r_{i, j}{ }^{p}$ $=\left[\left(\mathbf{K}_{z, i} / \epsilon_{i}\right)-\left(\mathbf{K}_{z, j} / \epsilon_{j}\right)\right] /\left[\left(\mathbf{K}_{z, i} / \epsilon_{i}\right)+\left(\mathbf{K}_{z, j} / \epsilon_{j}\right)\right]$ are the $p$-polarization amplitude reflection coefficients between layers $i$ and $j$, the $\mathbf{K}$ s are the wave vector components of the incident light normal to each layer, $\epsilon_{j}$ are the permittivities of the $j$ th layer, $n_{m}$ is the metal index, $n_{s}$ is the index of the surrounding medium, and $n_{2}$ is the index of the fiber cladding (for details, see [9]). The polarization dependence is simplistically incorporated into the Fresnel's equations by the introduction of $\sin (\delta)[13,14]$ AQ: References 13 to 16 have been renumbered sequentially in text. Please verify all edits into the $p$-polarized electric field component ( $\delta=\pi / 2$ is for $p$-polarized light and $\delta=0$ for $s$-polarized light), which determines the amplitude reflection coefficients [9].

The leaky $\mathrm{TE}_{v} / \mathrm{TM}_{v}$ modes' propagation constants are calculated using a dispersion relationship derived in [15] using the conformal mapping technique; they are obtained by solving expression (4) for the propagation constants, $\beta$, of the $\mathrm{TM}_{v}$ modes [Eq. (4a)] and $\mathrm{TE}_{v}$ modes [Eq. (4b)]:

$$
\begin{gathered}
\left(\frac{J_{v}{ }^{\prime}\left(u_{1} r_{1}\right)}{u_{1} r \cdot J_{v}\left(u_{1} r_{1}\right)} \cdot P_{v}+s_{21} \cdot \frac{Q_{v}}{W_{2}}\right) \cdot\left(\frac{K_{v}{ }^{\prime}\left(w_{3} r_{2}\right)}{w_{3} r_{2} \cdot K_{v}\left(w_{3} r_{2}\right)}\right. \\
\left.-s_{23} \frac{R_{v}}{\alpha_{2} \cdot W_{2}}\right)=\left(\frac{n_{2}{ }^{2}}{n_{1} n_{3} \alpha_{2} W_{2}{ }^{2}}\right)^{2}
\end{gathered}
$$

and

$$
\begin{aligned}
& \left(\frac{J_{v}{ }^{\prime}\left(u_{1} r_{1}\right)}{u_{1} r \cdot J_{v}\left(u_{1} r_{1}\right)} \cdot P_{v}+\frac{Q_{v}}{W_{2}}\right) \cdot\left(\frac{K_{v}{ }^{\prime}\left(w_{3} r_{2}\right)}{w_{3} r_{2} \cdot K_{v}\left(w_{3} r_{2}\right)}-\frac{R_{v}}{\alpha_{2} \cdot W_{2}}\right) \\
& \quad=\left(\frac{1}{\alpha_{2} W_{2}^{2}}\right)^{2}
\end{aligned}
$$

where phase parameters $u_{1}^{2}=\left(k^{2} n_{1}{ }^{2}-\beta^{2}\right)\left(1-x_{1} / r_{2}\right), w_{2}{ }^{2}$ $=\left(\beta^{2}-k^{2} n_{2}^{2}\right)\left(1-x_{1} / r_{2}\right)=W_{2}^{2} / r_{1}^{2}$, and $w_{3}^{2}=\left(\beta^{2}-k^{2} n_{3}^{2}\right)(1$ $\left.-x_{1} / r_{2}\right)=W_{3}^{2} / r_{2}{ }^{2}$ with the variables being $k$ as the wave number, $n_{1}$ as the refractive index of the core, $n_{2}$ as the cladding index, and $n_{3}$ as the index of the metal (silver). Also the functions $J$ and $K$ are Bessel functions of first kind and modified second kind, respectively, and $Q_{v}, P_{v}$, and $R_{v}$ are Bessel function cross-products (see [15] for more details). The parameters $r_{1}$ and $r_{2}$ are radii of the two concentric circles on an alternative $\zeta$-plane after the Mobius transform $z=r_{2}\left(z-x_{1}\right) /\left(z-x_{2}\right)$ with $x_{1}=R_{1}+d$ $-\sqrt{\left(2 R_{1} d+d^{2}\right)}, \quad x_{2}=R_{1}+d+\sqrt{\left(2 R_{1} d+d^{2}\right)}=r_{2}, \quad$ and $r_{1}=R_{1}$, where $d$ is the separation between the core-cladding interface and the metal surface and $R_{1}$ is the core radius, more details of the technique are given in [15]. The method adopted to solve expression (4) is a zoom search approach, the left-hand side of expression (1) is evaluated for ranges of real $\beta$ s and values of $\beta$ are chosen such that the left-hand sides of expression (4) are minimized or zero. Following this stage, it is repeated for a range of imaginary $\beta$ values for a given real $\beta$ solution. This approach yields the propagation constants of the coated D-shaped fiber leaky $\mathrm{TM}_{v}$ cladding modes.

The next step is the formulation of the field components of the $\mathrm{TE}_{v} / \mathrm{TM}_{v}$ modes of the fiber in order to calculate the coupling constants between the core and the cladding modes. This calculation is achieved by using the Debye potential functions (derived from the Helmholtz wave 
equation), along with the calculated $\beta$ s from expression (4) while implementing the boundary continuity conditions of this optical fiber along with normalization of the electric fields (E-fields). Adopting this approach leads to expressions (5)-(7), which are the nonzero field components of the $\mathrm{TM}_{v}$ cladding modes given in polar coordinates $(r, \phi, s)$ with $s$ being along the axis of the fiber:

$$
\begin{aligned}
E_{r}{ }^{\mathrm{cl}}= & \frac{\beta \cdot \mu_{1} A_{1} \Psi_{1}}{\omega \cdot w_{2} \epsilon_{1} \Psi_{2}} \cdot\left[I_{\nu}{ }^{\prime}\left(w_{2} r\right)-\left(\frac{\Psi_{2} \epsilon_{1} w_{2}}{\Psi_{1} \epsilon_{2}} \cdot J_{\nu}{ }^{\prime}\left(u_{1} r_{1}\right)\right.\right. \\
& \left.\left.+I_{\nu}{ }^{\prime}\left(w_{2}, r_{1}\right)\right) \frac{K_{\nu}{ }^{\prime}\left(w_{2} r\right)}{K_{\nu}{ }^{\prime}\left(w_{2} r_{1}\right)}\right], \\
H_{\phi}{ }^{\mathrm{cl}}= & \frac{u_{1} A_{1} \epsilon_{2} \Psi_{1}}{w_{2}{ }^{2} \epsilon_{1} \Psi_{2}} \cdot\left[I_{\nu}{ }^{\prime}\left(w_{2} r\right)-\left(\frac{\Psi_{2} \epsilon_{1} w_{2}}{\Psi_{1} \epsilon_{2}} \cdot J_{\nu}{ }^{\prime}\left(u_{1} r_{1}\right)\right.\right. \\
& \left.\left.+I_{\nu}{ }^{\prime}\left(w_{2} r_{1}\right)\right) \frac{K_{\nu}{ }^{\prime}\left(w_{2} r\right)}{K_{\nu}{ }^{\prime}\left(w_{2} r_{1}\right)}\right],
\end{aligned}
$$

and

$$
\begin{aligned}
E_{s}{ }^{\mathrm{cl}}= & \frac{u_{1} A_{1} \Psi_{1}}{i \omega \epsilon_{1} \Psi_{2}} \cdot\left[I_{\nu}{ }^{\prime}\left(w_{2} r\right)-\left(\frac{\Psi_{2} \epsilon_{1} w_{2}}{\Psi_{1} \epsilon_{2}} \cdot J_{\nu}{ }^{\prime}\left(u_{1} r_{1}\right)\right.\right. \\
& \left.\left.+I_{\nu}{ }^{\prime}\left(w_{2} r_{1}\right)\right) \frac{K_{\nu}{ }^{\prime}\left(w_{2} r\right)}{K_{\nu}{ }^{\prime}\left(w_{2} r_{1}\right)}\right] .
\end{aligned}
$$

Here $\Psi_{1}=u_{1} J_{\nu}\left(u_{1} r_{1}\right) K_{\nu}{ }^{\prime}\left(w_{2} r_{1}\right)-w_{2} J_{\nu}{ }^{\prime}\left(u_{1} r_{1}\right) K_{\nu}\left(w_{2} r_{1}\right)$ and $\Psi_{2}=K_{\nu}\left(w_{2} r_{1}\right) I_{\nu}{ }^{\prime}\left(w_{2} r_{1}\right)-K_{\nu}{ }^{\prime}\left(w_{2} r_{1}\right) I_{\nu}\left(w_{2} r_{1}\right)$, where $J_{\nu}\left(u_{1} r_{1}\right)$ is the Bessel function of the first kind of order $\nu . K_{\nu}\left(w_{2} r_{1}\right)$ and $I_{\nu}\left(w_{2} r_{1}\right)$ are modified Bessel functions of the first and second kind, respectively. $A_{1}$ is the field normalization constant with $\epsilon_{1}, \epsilon_{2}$ being the permittivity of the core and cladding, respectively.

Third, we calculate the individual coupling constants for each cladding mode from the core mode to confirm which leaky cladding modes are most effectively being coupled also by the TFBG, only the most significant being included in further calculations. This is achieved by evaluating the coupling coefficients derived in [13] using

$$
k_{\mathrm{cl}-\mathrm{co}}=\int_{0}^{2 \pi} \int_{0}^{r_{1}} E_{\mathrm{co}} \cdot \bar{E}_{\mathrm{cl}} \exp \left(i K_{t} \sin (\phi) r \mathrm{~d} r \mathrm{~d} \phi,\right.
$$

where $E_{\text {co }}$ is the E-field of the core mode and $E_{\text {cl }}$ is the E-field of the cladding mode. $K_{t}$ is the transverse wave number of the tilted grating, which gives the grating wave vector along the fiber axis; $K_{t}=-2 \pi \sin (\theta) / \Lambda$, where $\Lambda$ is the period of the grating and $\theta$ is the angle of tilt of the grating. The E-field components of the core and cladding modes are described in a cylindrical polar coordinate system, with the cladding modes given in expression (5) and the core mode expressed using the $\mathrm{LP}_{01}$ representation with polarization dependency given by [15]

$$
\begin{aligned}
E_{\mathrm{co}}= & J_{0}\left(u_{1} r_{1}\right) \cos (\phi-\delta) \cdot \hat{r}-J_{0}\left(u_{1} r_{1}\right) \sin (\phi-\delta) \cdot \hat{\phi} \\
& +\frac{i u_{1}}{\beta_{\mathrm{co}}} J_{1}\left(u_{1} r_{1}\right) \cos (\phi-\delta) \cdot \hat{s},
\end{aligned}
$$

where $\delta$ is the polarization angle with respect to the $x$ axis of the fiber (parallel to the flat of the D and normal to the fiber axis), once again $\delta=0^{\circ}$ represents $s$-polarized light and $\delta=90^{\circ}$ is $p$-polarized light with the cladding mode fields described by expressions (5)-(7).

\section{DISCUSSION}

First, it was found that the TFBG coupled to higher order $\mathrm{TM}_{\eta, v}$ modes in a D-shaped fiber than would be the case for a multimode or single-mode circular cross-sectional fiber, thus producing a larger range of scattering angles. It was noticed that for $\eta>2$ the coupling coefficients became significantly less than those for the lower order TM modes and that for $v \geqslant 11$, again, the values for coupling coefficients dramatically decreased. The typical calculated values are shown in Fig. 7.

Note that it is inappropriate to compare these calculated values to coupling coefficients for normal SMF re-

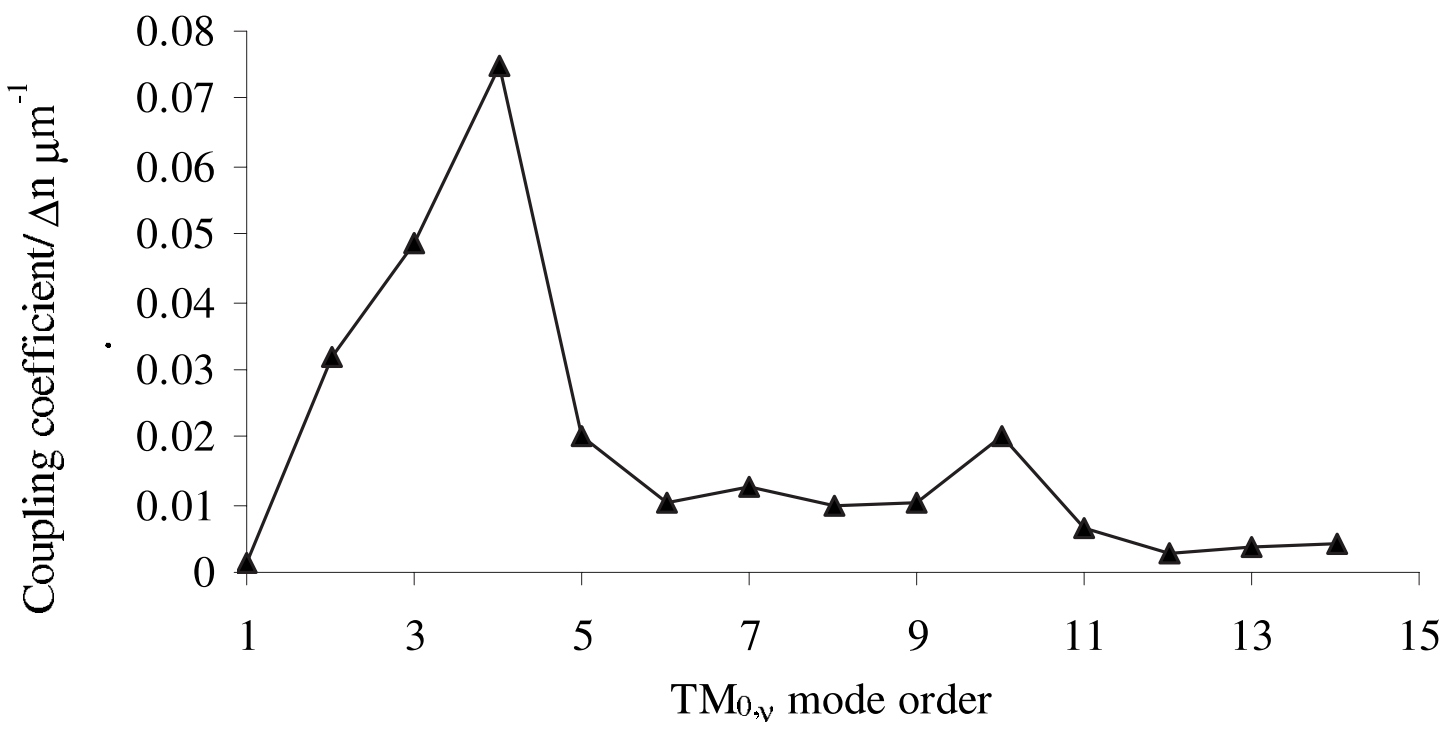

Fig. 7. Coupling coefficient for $\mathrm{TM}_{0, v}$ modes for a $7^{\circ}$ tilted grating in a D-shaped fiber with a silver coated flat. 


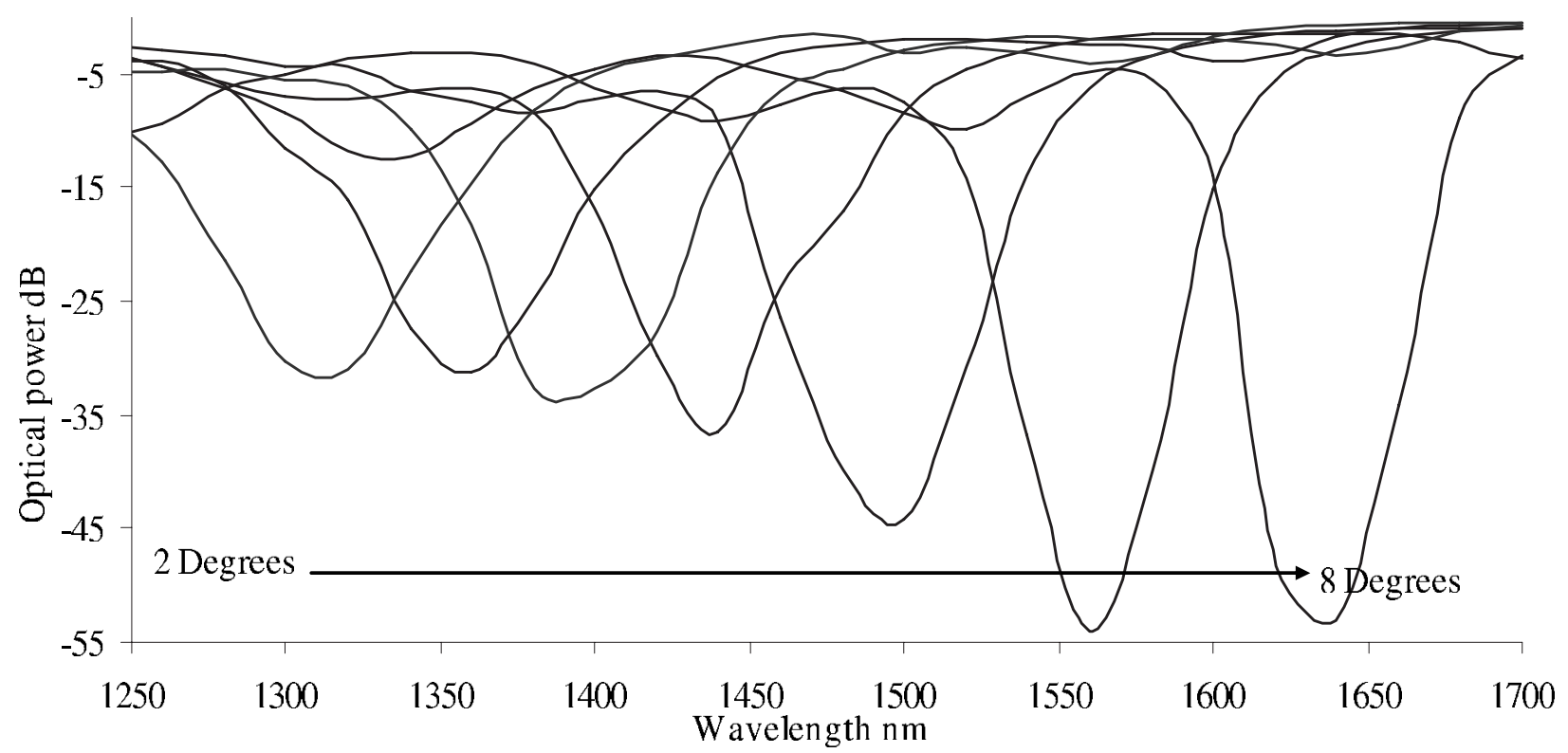

Fig. 8. Theoretically predicted transmission spectra of a SPR fiber device as a function of the azimuth, $\delta$, of the linearly polarized illuminating light (see text for other device parameters).

ported in literature [16], which are approximately 3 orders of magnitude greater, due to the fact that we are dealing with a metal coated D-shaped fiber with leaky cladding modes.

With the model described above it was possible to investigate the spectral dependence of coupling to the SP as a function of the polarization angle, $\delta$, for a linearly polarized input state. For a device with a $7^{\circ}$ tilted grating and $35 \mathrm{~nm}$ silver coating, the predicted transmission spectra of this SPR fiber device are shown in Fig. 8, with the spectral response and the coupling strength shown in Fig. 9. In both cases the shortest distance from the core/cladding interface of the fiber to the flat of the D was $8 \mu \mathrm{m}$ and the

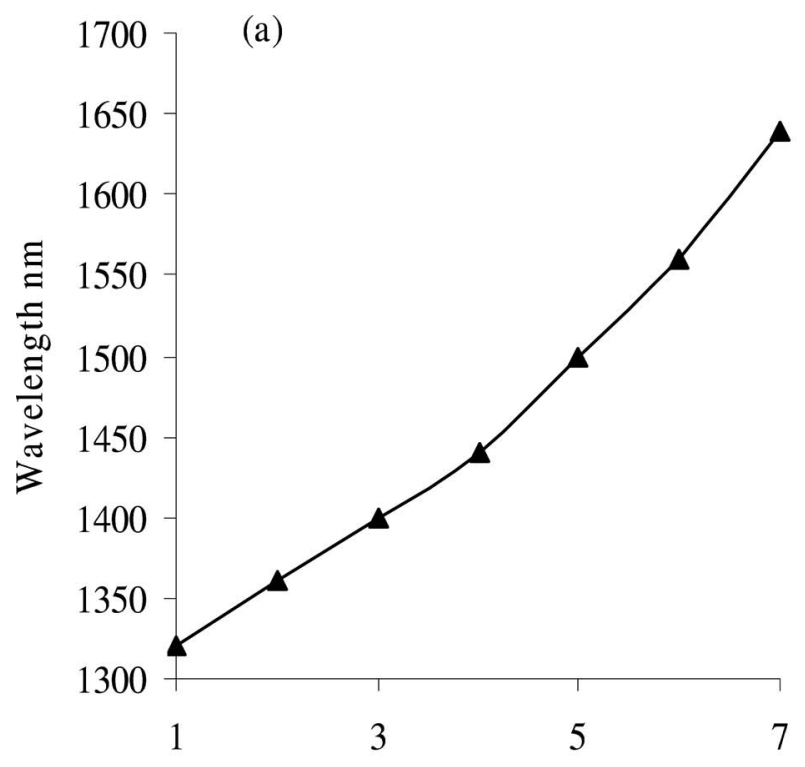

Change in $p$-polarization state degree fiber was assumed to be surrounded by a medium of refractive index 1.36. The $\delta$ valves were chosen to produce resonances in the same spectral region as those observed experimentally.

It was noticed during the spectral simulations that for a given refractive index of the surrounding medium, only a few TM modes contributed to the main spectral features of the device. In the case of a refractive index of 1.36 , these modes were $\operatorname{TM}_{0}(0,1), \mathrm{TM}_{1}(2,3)$, and $\mathrm{TM}_{2}(1,2,3)$; the net effect is a broadening of the resonance from what would be the case were there only one mode involved.

Comparing the experimental data in Fig. 3 to that in Fig. 8 shows that experimental broadening appears to be

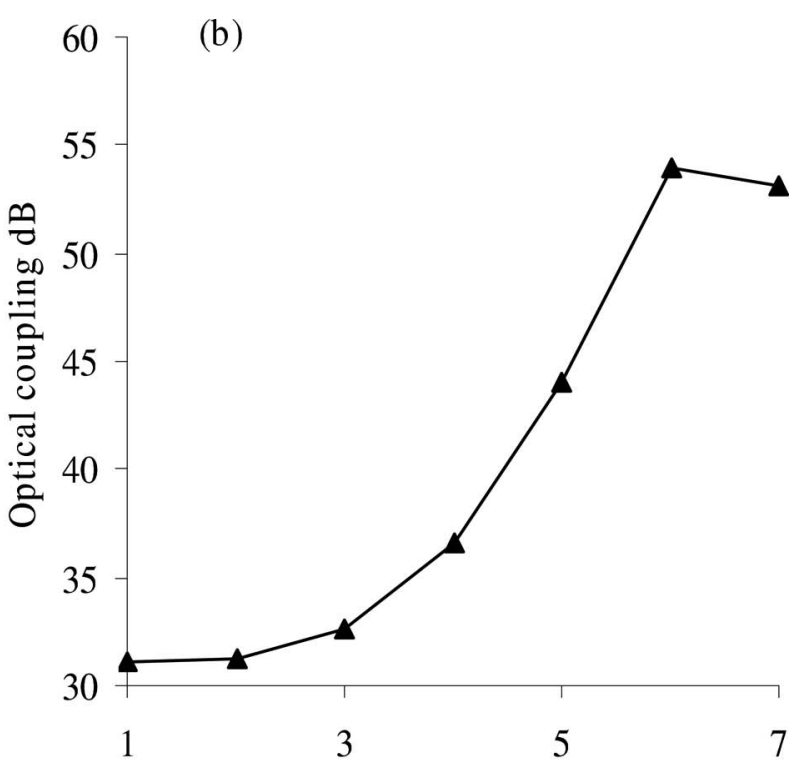

Change in $p$-polarization state degree

Fig. 9. Theoretically predicted spectral response (a) and coupling strength (b) of a SPR fiber device as a function of the azimuth of polarization of the illuminating linearly polarized light (see text for other device parameters). 


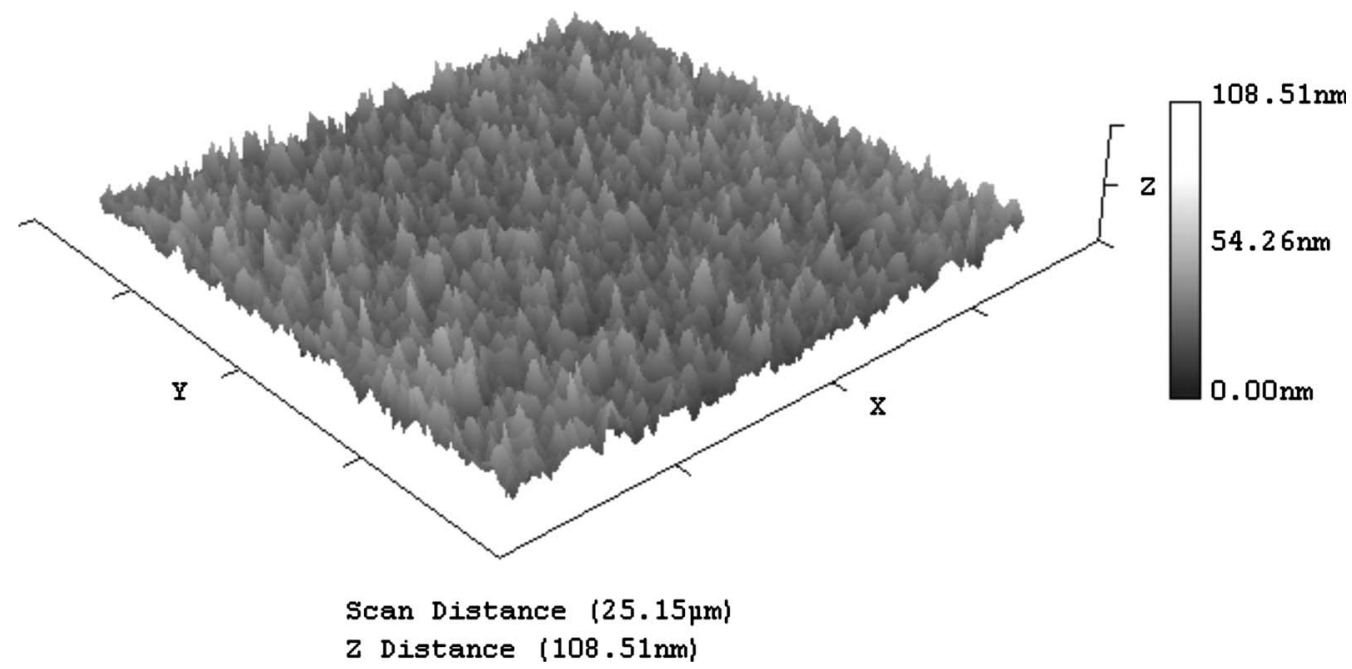

Fig. 10. Typical image of the surface of a silver coated D-shaped fiber taken with an AFM.

larger than that theoretically predicted; the observed FWHM is $\sim 350 \mathrm{~nm}$ compared to a FWHM of $\sim 100 \mathrm{~nm}$ from the model. To check that this discrepancy was not due to an inadequate amount of higher order TM modes, more modes were including in the calculation for the predicted transmission spectra, but it was found that this did not significantly increase the broadening of the resonances. Another possible explanation relates to surface roughness or nonuniformities of the silver film that has been sputtered onto the lapped region of the device. The surface roughness of the silver coatings were measured by an atomic force microscope (AFM) (Pacific Nanotechnology, Nano R); measurements were carried out on four coated samples with several scans per sample, totaling 12 all together and all showing similar results. Figures 10 and 11 show typical results obtained from the AFM.

By using the data obtained from the AFM in conjunction with the data analysis software NANORULE+ (Pacific Nanotechnology [17]) we found that samples of the sput- tered silver coating typically had an average step height/ thickness of $23 \mathrm{~nm}$. Also the measured average roughness of the silver coatings was $6 \mathrm{~nm}$ with the thickness ranging up to $58 \mathrm{~nm}$. This variation of thickness and roughness of coating will have an effect on the SP generated in the wavelength range from 1000 to $1700 \mathrm{~nm}$ due to the fact that the skin depth of silver in this spectral regime is around $10 \mathrm{~nm}$.

The granularity dimensions of the silver varied in scale from $\sim 0.1$ to $2 \mu \mathrm{m}$ with an average of $\sim 0.8 \mu \mathrm{m}$, see Fig. 11. A consequence of the physical properties of the coatings is that the SPs that are generated may have short propagation lengths compared to typical values from smooth surfaces [4], which range from 50 to $150 \mu \mathrm{m}$ and have a resonance FWHM of just a few nanometers compared to the hundreds of nanometers seen with our device. Further work is clearly required to explain this behavior.

In comparing Figs. 4 and 9 displaying the spectral tun-
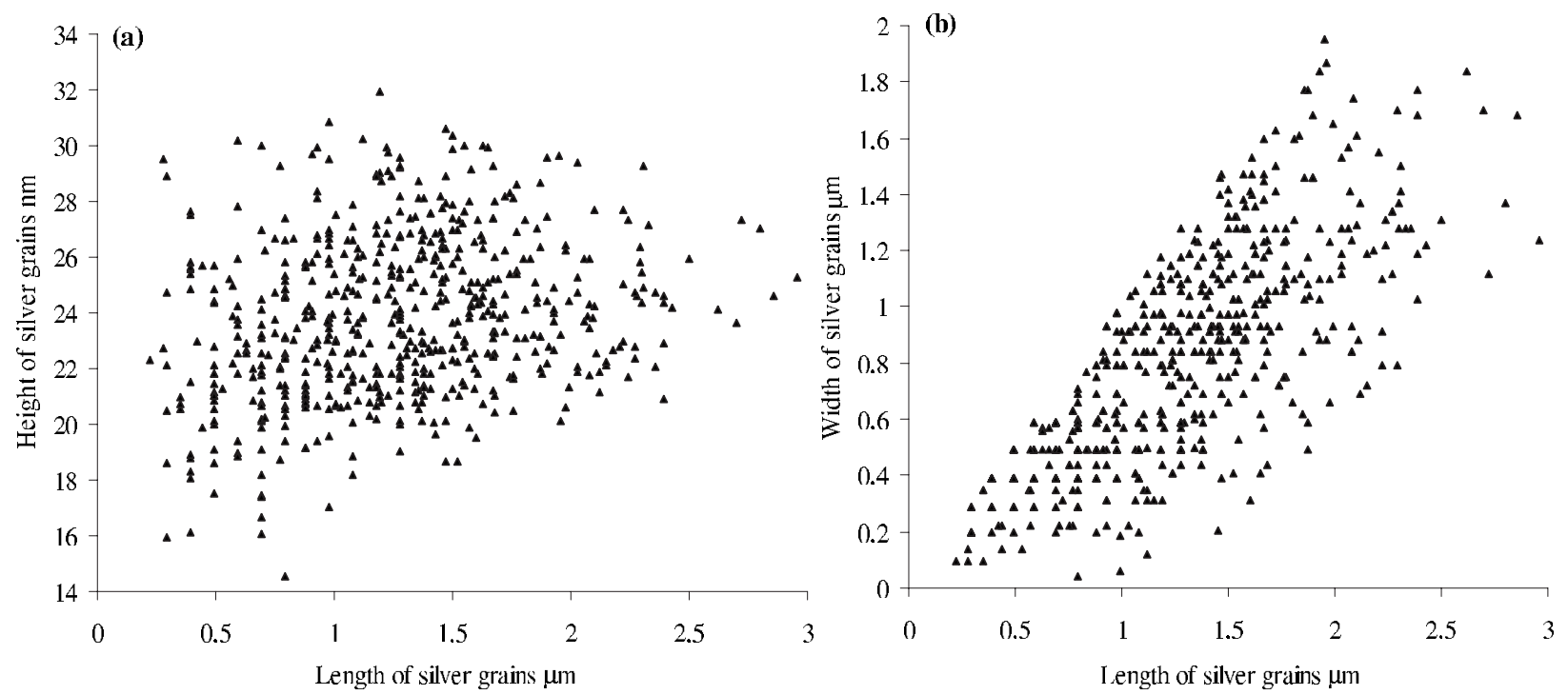

Fig. 11. Grain analysis of a typical silver coating on the D-shaped fiber: (a) scatter plot of height of the grains against length of the grains, and (b) scatter plot of width of the grains against length of the grains. 


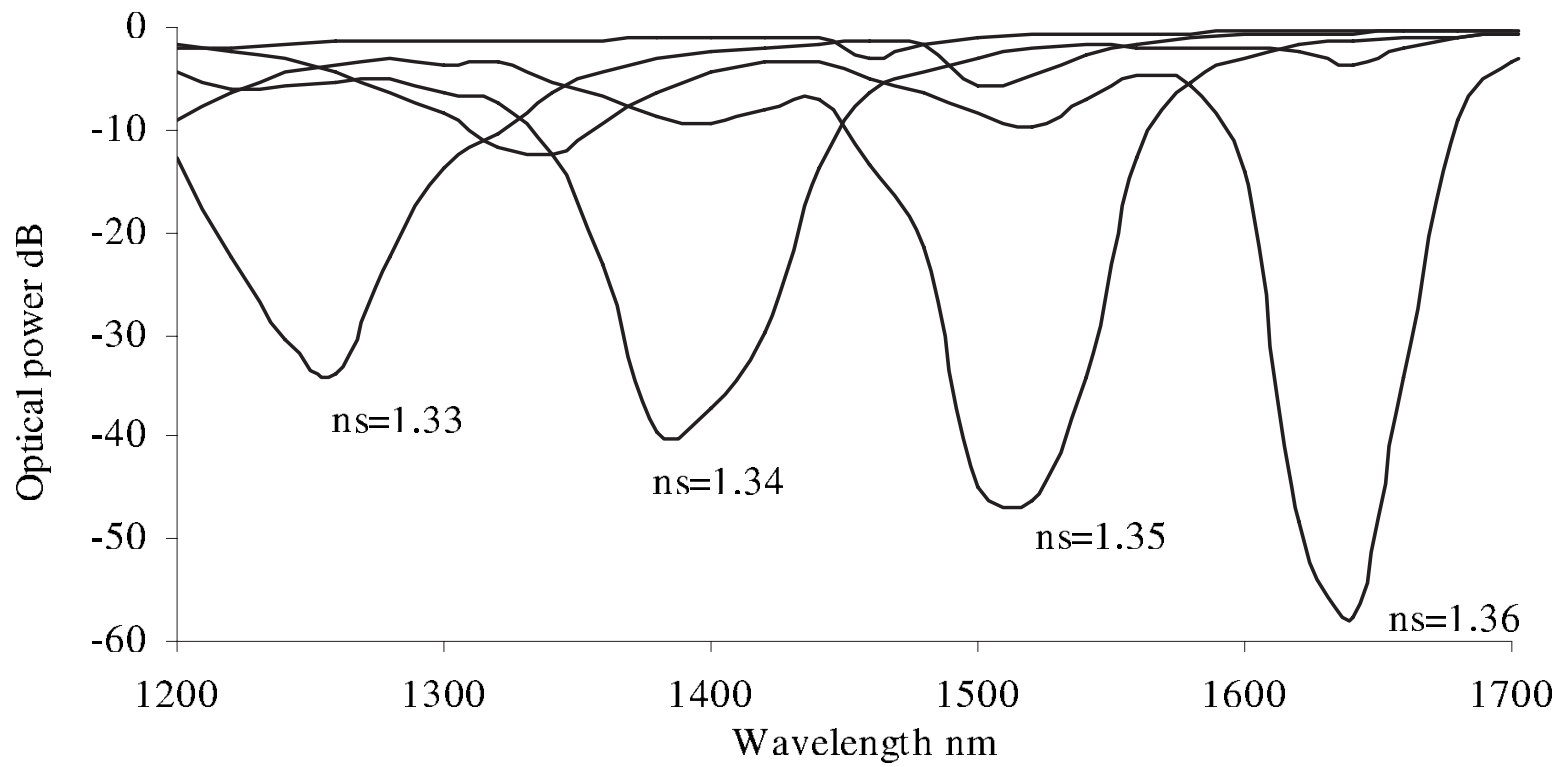

Fig. 12. Theoretical transmission spectra as a function of the surrounding medium's refractive index for a SPR fiber device for $p$-polarization state of the illuminating light. The device has a tilt angle of $7^{\circ}$ and a $35 \mathrm{~nm}$ coating of silver.

ability and coupling strength of the SPR as a function of polarization; the experimental data in Fig. 4 show a higher sensitivity to the polarization of the illuminating light than the theoretical data in Fig. 9. This may be due to the fact that the lapped fiber may not be a perfect D-shape but possess asymmetric geometrical features, which could cause greater polarization dependence. The predicted coupling strength is much higher than experimentally observed, which is expected because roughness was not included in the model of the SPR device.

Further calculations to reproduce the transmission spectra of the devices suggest that for different polarization states, SPRs are being generated from the same spatial regions with different resonant wavelengths. This is important as it suggests that the spatial extension of the evanescent fields of the SP into the surrounding medium at a given spatial location could be controlled via the polarization of the illuminating light. This controllability of the SP could be used to provide a degree of depth resolution when interrogating thicker films deposited on the silver coating.

The model was also used to predict the spectral behavior as a function of the surrounding medium's refractive index. Figure 12 shows an example of the theoretically predicted transmission as a function of index, and the spectral response and coupling strength are shown in Fig. 13. By comparing the theoretically predicted behavior to the experimental data shows some differences, which may be expected due to the lack of reproducibility in the current fabrication process but the same general trends, which is to be expected given that the model represents an idealized case with purely $p$-polarized light and no surface roughness. The modeling suggests that under optimum fabrication and working conditions for a $7^{\circ}$ tilted grating device, an index sensitivity of $\mathrm{d} \lambda / \mathrm{d} n \sim 18,000 \mathrm{~nm}$ could be achieved, leading to a resolution (under the as-
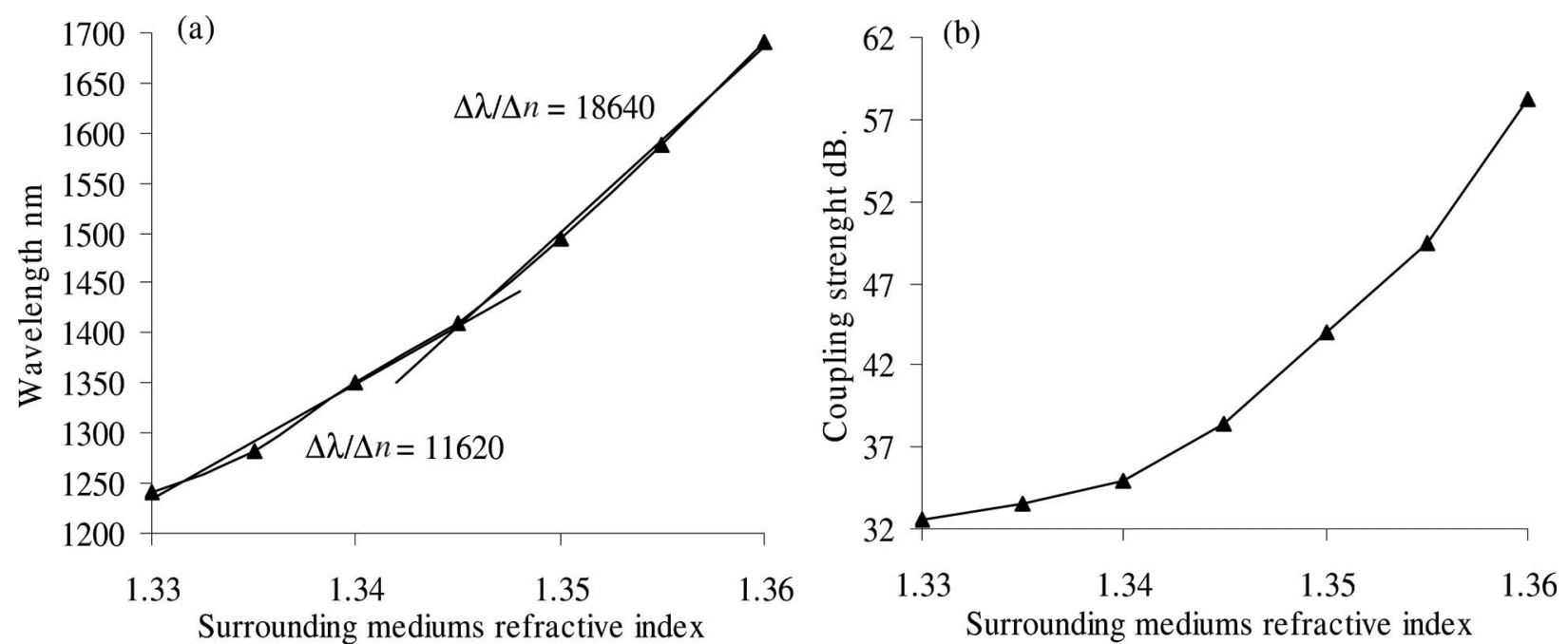

Fig. 13. Theoretically predicted spectral response (a) and coupling strength (b) of a SPR fiber device as a function of the surrounding medium's refractive index for $p$-polarization state of the illuminating light. The device has a tilt angle of $7^{\circ}$ and a $35 \mathrm{~nm}$ coating of silver. 
sumption of a $0.1 \mathrm{~nm}$ measurement resolution for the resonance wavelength) of $\sim 5 \times 10^{-6}$ over the index range from 1.34 to 1.37 .

\section{CONCLUSION}

We have demonstrated what we believe to be a novel SPR fiber device utilizing a TFBG to enhance the coupling of the illuminating light to localized SPs on a silver coated lapped single-mode fiber.

It was found that by altering the polarization of the light the resonance could be tuned over the spectral range from 1100 to $1700 \mathrm{~nm}$ with extinction ratios in excess of $35 \mathrm{~dB}$ for a surrounding medium in the aqueous index regime (from 1.34 to 1.37). The polarization dependence can also control the spatial extension of the SPR into the surrounding medium at a given spatial location. The variations in optical characteristics found with the same angle of TFBG in different devices suggests that a more repeatable fabrication procedure needs to be implemented. A simple model was produced, which showed reasonable agreement with the experimental data with regard to polarization dependence and refractive index sensitivity.

\section{REFERENCES}

1. S. Vasilev and O. Medvedkov, "Long-period refractive index fibre gratings: properties, applications and fabrication techniques," Proc. SPIE 4083, 212-223 (2000).

2. S.-M. Tseng, K.-Y. Hsu, H.-S. Wei, and K.-F. Chen, "Analysis and experiment of thin metal-clad fiber polarizer with index overlay," IEEE Photon. Technol. Lett. 9, 628-630 (1997).

3. K. Schroeder, W. Ecke, R. Mueller, R. Willsch, and A. Andreev, "A fibre Bragg grating refractometer," Meas. Sci. Technol. 12, 757-764 (2001).

4. M. Piliarik, J. Homola, Z. Maníková, and J. Ctyroký,
"Surface plasmon resonance sensor based on a single-mode polarisation-maintaining optical fiber," Sens. Actuators B 90, 236-242 (2003).

5. J. Homola, S. Yee, and G. Gauglitz, "Surface plasmon resonance sensors: review," Sens. Actuators B 54, 3-15 (1999).

6. S. Patskovsky, A. Kabashin, M. Meunier, and J. Luong, "Properties and sensing characteristics of surface plasmon resonance in infrared light," J. Opt. Soc. Am. A 20, 1644-1650 (2003).

7. T. Erdogan, "Fiber grating spectra," J. Lightwave Technol. 15, 1277-1292 (1997)

8. M. Iga, A. Seki, and K. Watanabe, "Gold thickness dependence of SPR-based hetero-core structured optical fiber sensor," Sens. Actuators B 106, 363-368 (2005).

9. H. Raether, ed., "Surface Plasmons on Smooth and Rough Surfaces and on Gratings," (Academic, 1997).

10. T. Allsop, R. Neal, S. Rehman, D. J. Webb, D. Mapps, and I. Bennion, "Surface plasmon resonance generation utilising tilted fiber Bragg grating for the biochemical sensing," in Proceedings of the OFS-18, Biological and Medical Sensors (2006), paper WA 4

11. T. Allsop, R. Neal, S. Rehman, D. J. Webb, D. Mapps, and I. Bennion, "The generation of infrared surface plasmon resonances with high refractive index sensitivity utilizing tilted fibre Bragg gratings,” Appl. Opt. 46, 5456-5460 (2007).

12. R. Walker, S. Mihailov, P. Lu, and D. Grobnic, "Shaping the radiation field of tilted fiber Bragg gratings," J. Opt. Soc. Am. B 22, 962-975 (2005)

13. A. Zayats and I. Smolyaninov, "Near-field photonics: surface plasmon polaritions and localized surface plasmons," J. Opt. A, Pure Appl. Opt. 5, 16-50 (2003).

14. J. Homola and S. Yee, "Novel polarisation control scheme for spectral surface plasmon resonance sensors," Sens. Actuators B 51, 331-339 (1998).

15. C. Tsao, ed., "Optical Fibre Waveguide Analysis" (Oxford, 1992).

16. K. S. Lee and T. Erdogan, "Fiber mode coupling in transmissive and reflective tilted fiber gratings," Appl. Opt. 39, 1394-1404 (2000).

17. NanoRule ${ }^{\mathrm{TM}}$, http://www.pacificnanotech.com/. 